\title{
Power-Free Microchip Immunoassay of PSA in Human Serum for Point-of-Care Testing
}

\author{
Hiroki OKada, Kazuo HosoKawa, ${ }^{\dagger}$ and Mizuo MaEda \\ Bioengineering Laboratory, RIKEN, 2-1 Hirosawa, Wako, Saitama 351-0198, Japan
}

\begin{abstract}
For point-of-care testing (POCT), a sandwich-typed immunoassay on poly(dimethylsiloxane) (PDMS) microfluidic chips was developed by employing two original technologies: a power-free microchip and laminar flow-assisted dendritic amplification (LFDA). Sequential injection of reagents of immunoassay was carried out by the power-free microchip. In addition, for the amplification of a fluorescent signal, LFDA exploited the interaction between FITC-labeled streptavidin and biotinylated anti-streptavidin on the interface of the two streams of laminar flow during the sequential injection of the reagents of the immunoassay. The microfluidic immunoassay was finished within $21 \mathrm{~min}$ with a sample volume of $500 \mathrm{~nL}$. The calibration curve of a quantitative analysis satisfied the concentration range of the cut-off values of prostate-specific antigen (PSA) in serum. The limit of detection (LOD) reached $520 \mathrm{pg} / \mathrm{mL}(16 \mathrm{pM})$ of PSA in human female serum. We believe that this microfluidic immunoassay and device are promising for POCT.
\end{abstract}

(Received November 26, 2010; Accepted January 24, 2011; Published March 10, 2011)

\section{Introduction}

The death rate from prostate cancer ( $\mathrm{PCa}$ ) is rapidly growing in Japan and PCa is the second leading cause of male cancer-related death in the United States. ${ }^{1,2}$ A diagnostic indicator of PCa is elevated levels of prostate-specific antigen (PSA): a 33-kDa glycoprotein, one of serine protease and a product of the prostate epithelium to maintain liquefaction of seminal fluid., ${ }^{2,3}$ Importantly, PSA is a most useful serum marker for the clinical screening and diagnosis of PCa. The upper threshold of the normal PSA level is generally utilized at the range from 4 to $10 \mathrm{ng} / \mathrm{mL}$. A serum concentration of PSA above the cut-off values increased the likelihood of PCa, and a biopsy was needed to confirm the diagnosis of PCa. ${ }^{3,4}$

The test of the serum PSA level is mainly executed in hospital laboratories or research institutes equipped with large, expensive and complex instruments requiring special technical skills to operate, so that both a low detection limit and an accurate analysis are ensured. The fact that PSA testing is performed at dedicated and centralized facilities requires transport of the sample to the testing site and a substantial time to evaluate data. This time interval retards the results of the testing results back to clinicians. Namely, this fact forces patients against such burdens as waste of time, catastrophic costs for diagnosis and clinical visits.

Point-of-care testing (POCT) is defined as the rapid molecular diagnosis of disease at or near the side of patient care. As innovative technologies progress, POCT is of growing importance in various clinical fields, such as routine health assessment, the progression of disease and the prediction of risk. The characteristics of POCT have potential to overcome such demerits for patients. ${ }^{5}$ Immunochromatographic strip tests,

$\dagger$ To whom correspondence should be addressed.

E-mail: k-hoso@riken.go.jp also known as immunostrips, have been developed as POCT of PSA due to their advantages, including low cost, ease of use, rapid analysis (about $10 \mathrm{~min}$ ), contamination free due to paper chromatographic separation of a sample and long-term stability over a wide range of climates. However, no immunostrips on marketplace have worked as a perfect alternative to centralized PSA testing because immunostrips depend on visual readout limiting sensitive and quantitative analysis. ${ }^{6,7}$ The present state of immunostrips for PSA testing allowed its application in forensic science rather than the diagnosis of $\mathrm{PCa}$, because forensic applications require only limited sensitivity. ${ }^{8,9}$ Therefore, advanced platforms for POCT with the advantages of being compact, low-cost, high sensitivity, quantitative and foolproof would be beneficial. ${ }^{10}$

Microfluidics has enabled many of the most dramatic developments, particularly in biology and chemistry, because it has excellent potential for fast analysis, even small analyte consumption and good integration of analytical procedures. Additionally, it often offers not only simple and extremely less-hazardous operation to use but also cost-effective and portable platforms. ${ }^{5}$ Therefore, these characteristics open up microfluidics as a promising device of POCT, and many efforts have been made. ${ }^{11-13}$ However, most of them were hooked up to an external pumping system, electric power supply ${ }^{11}$ and complicated fluidic designs. ${ }^{12,13}$ It is demanded to progress further in microfluidics with not only simpler and more compact device integrating the external power sources but also user-friendliness. Some research groups improved the microfluidic assays for POCT. For example, a capillary-driven microfluidic chip for an one-step immunoassay of C-reactive protein (CRP) was demonstrated. ${ }^{14}$ Multiplex biomarkers detection was performed using an immuno-pillar chip. ${ }^{15}$

Recently, our group developed "power-free microchip"16 by using poly(dimethylsiloxane) (PDMS), which has become one of the most widely used materials for microfluidics, primarily due to its straightforward way and low cost to fabricate. 
Additionally, PDMS possesses other important characteristic of gas permeability, which was skillfully turned into developing "power-free microchip". Specifically, inlets and outlets of the PDMS chip, preliminarily degassed in a vacuum chamber, were closed by reagents of immunoassay and tape, respectively. The entrapped air in the microchannel was automatically absorbed into PDMS, and as a result negative pressure was created in the channel and worked as a pumping system. A microfluidic immunoassay of CRP as a model analyte was demonstrated using the power-free microchip. ${ }^{17}$ Furthermore, laminar flow-assisted dendritic amplification (LFDA) was adopted to realize a highly sensitive detection of CRP. ${ }^{18}$ LFDA allowed the immunoreactions to take place in the interface of two laminar streams, and the fluorescent signal was amplified via the interaction between fluorophore-labeled streptavidin and biotinylated anti-streptavidin. As a result, LFDA improved the LOD of the CRP immunoassay on the power-free microchip by 3 orders of magnitude down to $17 \mathrm{pg} / \mathrm{mL}$. At that time, CRP was useful as a model antigen to evaluate the effect of LFDA. However, the ultrasensitive detection of CRP actually has little significance from a practical point of view, because the clinical cut-off value of CRP is relatively high $(3 \mu \mathrm{g} / \mathrm{mL}) .{ }^{18}$

In this paper, we describe the application of the power-free microchip immunoassay with LFDA to a more practical antigen, PSA, which has clinical cut-off values of 4 and $10 \mathrm{ng} / \mathrm{mL}$. The samples were prepared by diluting PSA with human female serum, which originally contained no PSA, to emulate clinical samples. Similarly to the CRP case, the use of LFDA improved the LOD by more than 2 orders of magnitude. With a sample volume of $500 \mathrm{~nL}$ and a detection time of $21 \mathrm{~min}$, we obtained a calibration curve that well covered the PSA cut-off values.

\section{Experimental}

\section{Device fabrication}

The fabrication procedure of microfluidic chips was described elsewhere. ${ }^{18}$ A $25-\mu \mathrm{m}$-thick negative photoresist (SU-8 25, MicroChem, Newton, MA) was spin-coated onto a silicon wafer. After UV exposure and development, a silicon mold with Y-shaped fluidic patterns was obtained for the fabrication of PDMS-based microchannels. Before fabrication of the channels, the mold was exposed to $\mathrm{CHF}_{3}$ plasma in a plasma etcher (RIE-10NR, Samco International, Kyoto, Japan) for $10 \mathrm{~s}$. For the casting of PDMS, the precursor prepared by mixing prepolymer of PDMS with the curing agent from Sylgard 184 kit (Dow Corning, Midland, MI) at a weight ratio of 10:1 was poured onto the mold with a plastic frame for holding the prepolymer. The cured PDMS $(30 \times 30 \times 2 \mathrm{~mm})$ was peeled off from the mold and was partially cut for open inlets. Waste reservoirs connecting to the channels were formed using a metal punch. The PDMS with the Y-shaped design $(100 \mu \mathrm{m}$ wide and $25 \mu \mathrm{m}$ deep microchannel) was reversibly bonded to a flat PDMS $(30 \times 30 \times 2 \mathrm{~mm})$ slab, which had been obtained by the same molding procedure as mentioned above.

\section{Sample and reagent preparation}

To simulate a clinical condition, the immunoassay of PSA diluted in human female serum was carried out, and the results were compared with data obtained with PSA diluted in phosphate-buffered saline (PBS). Sample solutions of PSA were created by diluting PSA (Acris Antibody, Herford, Germany) either in PBS (pH 7.5, Cambrex Biosciences, Walkersville, MD) or in human female serum (Scipac, Kent, UK) to the desired concentrations. The serum was used without centrifugation. The solution of a capture antibody (c-Ab) contained $500 \mu \mathrm{g} / \mathrm{mL}$ mouse anti-human PSA (Funakoshi, Tokyo, Japan) in PBS. Biotinylated goat anti-human PSA (R\&D Systems, Minneapolis, MN) as a biotinylated antibody (b-Ab) was diluted with $1 \%(w / v)$ bovine serum albumin (BSA, Wako Pure Chemical Industries, Osaka, Japan) in PBS at a concentration of $75 \mu \mathrm{g} / \mathrm{mL}$. Solutions of FITC-labeled streptavidin (F-SA, Sigma, St. Louis, MO) and biotinylated anti-streptavidin (b-anti-SA, Rockland Immunochemicals, Gilbertsville, PA) were created using $1 \%$ BSA in PBS at concentrations of 7.5 and $75 \mu \mathrm{g} / \mathrm{mL}$, respectively. Powder of the blocking reagent (Roche Diagnostic, Tokyo, Japan) was dissolved in $1 \%$ BSA in PBS at a concentration of $0.1 \%(\mathrm{w} / \mathrm{v})$.

\section{Immunoassay}

Immunoassays were performed using both the power-free sequential injection and the LFDA method. ${ }^{18}$ Prior to immunoassay, air dissolved in the PDMS microchip was removed in a vacuum desiccator at $10 \mathrm{kPa}$ for $1 \mathrm{~h}$. The microchip was taken out from the desiccator, and soon afterwards the upper face of the chip (the face containing waste reservoirs) was covered up with a piece of adhesive tape (Sumitomo 3M, Tokyo, Japan). A $0.5-3.5 \mu \mathrm{L}$ aliquot of the solution of the immunoassay to be injected was dispensed at the inlet opening with a micropipette. The following solutions were sequentially injected: (1) $0.5 \mu \mathrm{L}$ of c-Ab from both the left and right inlets ( $\mathrm{R}$ and $\mathrm{L}$, respectively) in order to physically adsorb $\mathrm{c}-\mathrm{Ab}$ onto the inner surfaces of the microchannels for $5 \mathrm{~min}$, (2) $0.5 \mu \mathrm{L}$ of blocking reagent from both $\mathrm{R}$ and $\mathrm{L}$ for $4 \mathrm{~min}$, (3) $0.5 \mu \mathrm{L}$ of PSA through $\mathrm{L}$ and $0.5 \mu \mathrm{L}$ of b-Ab through $\mathrm{R}$ for $5 \mathrm{~min}$, (4) $3.5 \mu \mathrm{L}$ of F-SA through $\mathrm{L}$ and $3.5 \mu \mathrm{L}$ of b-anti-SA through R. As negative control experiments of LFDA, 1\% BSA in PBS was used as a substitute for the b-anti-SA solution.

\section{Data capture and analysis}

Fluorescent measurements were carried out using an inverted fluorescence microscope (TE2000-U, Nikon, Tokyo, Japan) equipped with a $100 \mathrm{~W}$ mercury lamp, a dichroic mirror for excitation (465 - $495 \mathrm{~nm})$ and emission $(515-555 \mathrm{~nm})$, a $10 \times$ objective lens, a cooled CCD camera (CoolSNAP HQ2, Photometrics, AZ) and the software (QCapture Pro 6.0, Media Cybernetics, MD) for acquiring and collecting image data. The exposure time $(t)$ of the CCD camera was varied between $100 \mathrm{~ms}$ and $1000 \mathrm{~ms}$, depending on the brightness of obtained images. The mean fluorescent intensity $(y)$ in the region of interest (dot line in Fig. 1a) was collected from the region using Image J 1.42q (National Institute of Health, USA) and determined by the following equation: $y=\left(f-f_{0}\right) / t$, where $f$ and $f_{0}$ denote brightness of the image in the two flow streams and in outside of the microchannel (Fig. 1b), to obtain the time course and a dose-response curve for the immunoassay. The data was fitted with the four-parameter logistic model equation: ${ }^{19} y=f(x ; a, b$, $c, \quad d)=d+(a-d) /\left[1+(x / c)^{\mathrm{b}}\right]$, where $x$ denotes that the concentration of PSA, and $a, b, c$ and $d$ are the curve-fitting parameters. These parameters were calculated by a nonlinear least-square method with the weight of reciprocal of the variance of each data value using graphing software (Kaleida Graph 3.51, Hulinks, Tokyo, Japan), and utilized to determine the limit of detection (LOD). The LOD was defined as the lowest concentration for which the background-subtracted fluorescence signal was greater than three standard deviations $(3 \sigma)$ above the average signal of the blank sample. 

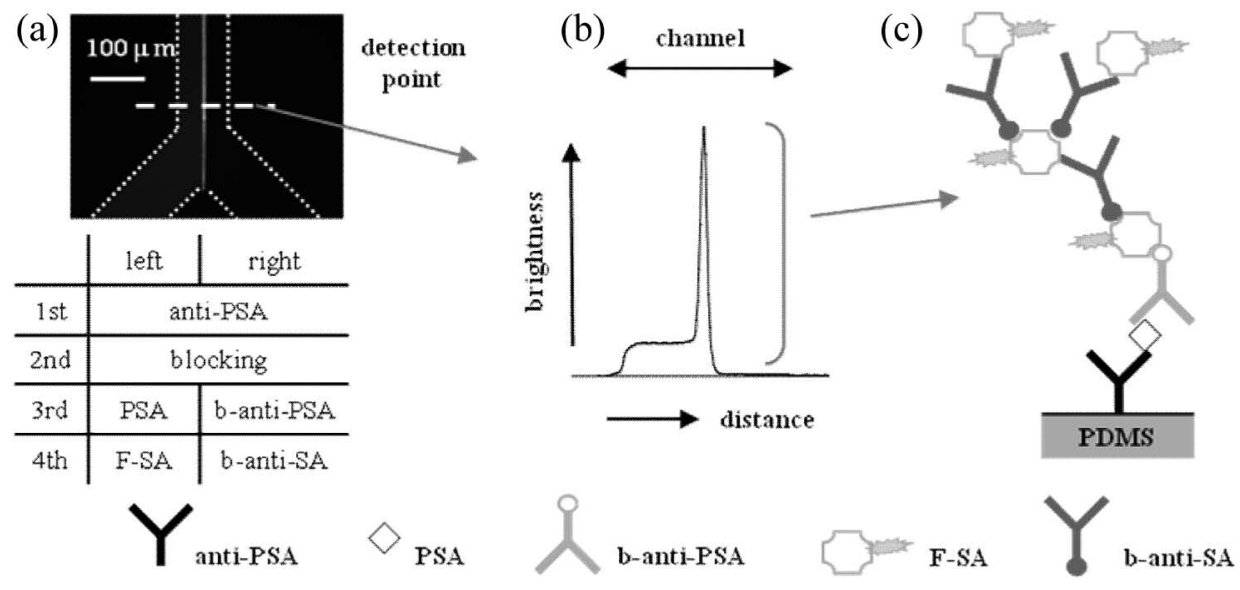

Fig. 1 (a) Procedure of immunoassay and fluorescent image around the detection point. (b) Fluorescent profile at the detection point. (c) Schematic of LFDA. Fluorescence is amplified by the biotin-avidin interaction.

$(\mathrm{a}-1)$

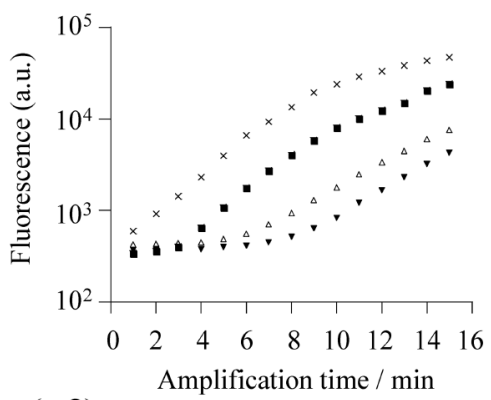

(a-2)

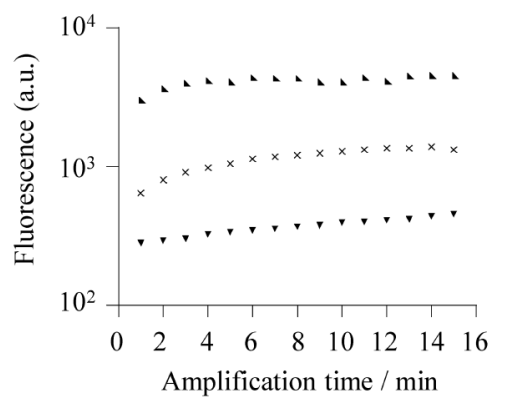

(b-1)

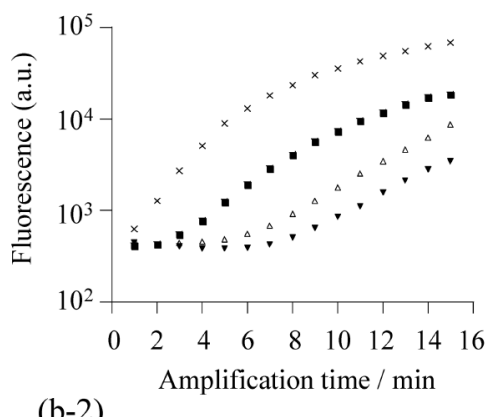

(b-2)

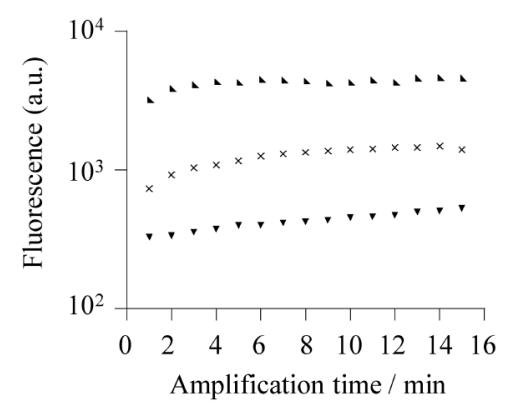

Fig. 2 Time course of the fluorescent intensity of the microfluidic immunoassay. The $0 \mathrm{~min}$ of amplification time in the horizontal axis is defined as the time from which both F-SA and b-anti-SA start flowing into the microchannel. PSA was diluted with PBS containing 1\% BSA (a-1 and a-2) or human female serum (b-1 and b-2). Each data point is the average of four measurements obtained by four independent experiments. Additionally, fluorescence was obtained from LFDA (a-1 and b-1) or from no amplification (a-2 and b-2). The concentrations of PSA: blank $(\boldsymbol{\nabla}), 4.0 \mathrm{ng} / \mathrm{mL}(\triangle), 100 \mathrm{ng} / \mathrm{mL}$ (ם), $1.0 \mu \mathrm{g} / \mathrm{mL}(\times)$, and $10 \mu \mathrm{g} / \mathrm{mL}(\mathbf{\Delta})$.

\section{Results and Discussion}

\section{Time course of fluorescent intensity}

As described in the experimental section, the reagents of the microfluidic immunoassay (MIA) were sequentially injected into a Y-shaped microchannel, and the reagents of MIA were mixed and reacted at the interface of two streams of laminar flow, resulting in forming a sandwich-typed structure in the interface (Fig. 1c). Unlike sandwich-typed ELISA, biotinylated antibody of PSA (b-Ab) was the alternative of the enzyme-conjugated antibody, and worked as the origin of LFDA. The exponential binding of both F-SA and b-anti-SA was started, and the fluorescent signal was enhanced at the interface via both the biotin-avidin and the antigen-antibody interactions over time. The flow rate was passively determined by power-free microchip, ${ }^{16-18}$ and it was estimated from the volume and the flowing time of the aliquots; 1.3 to $1.7 \mathrm{~mm} / \mathrm{s}$.

The time course of the amplified signal by LFDA is shown in Figs. 2a-1 and 2b-1. To check the effect of human female serum, MIA was performed using two kinds of PSA samples: 

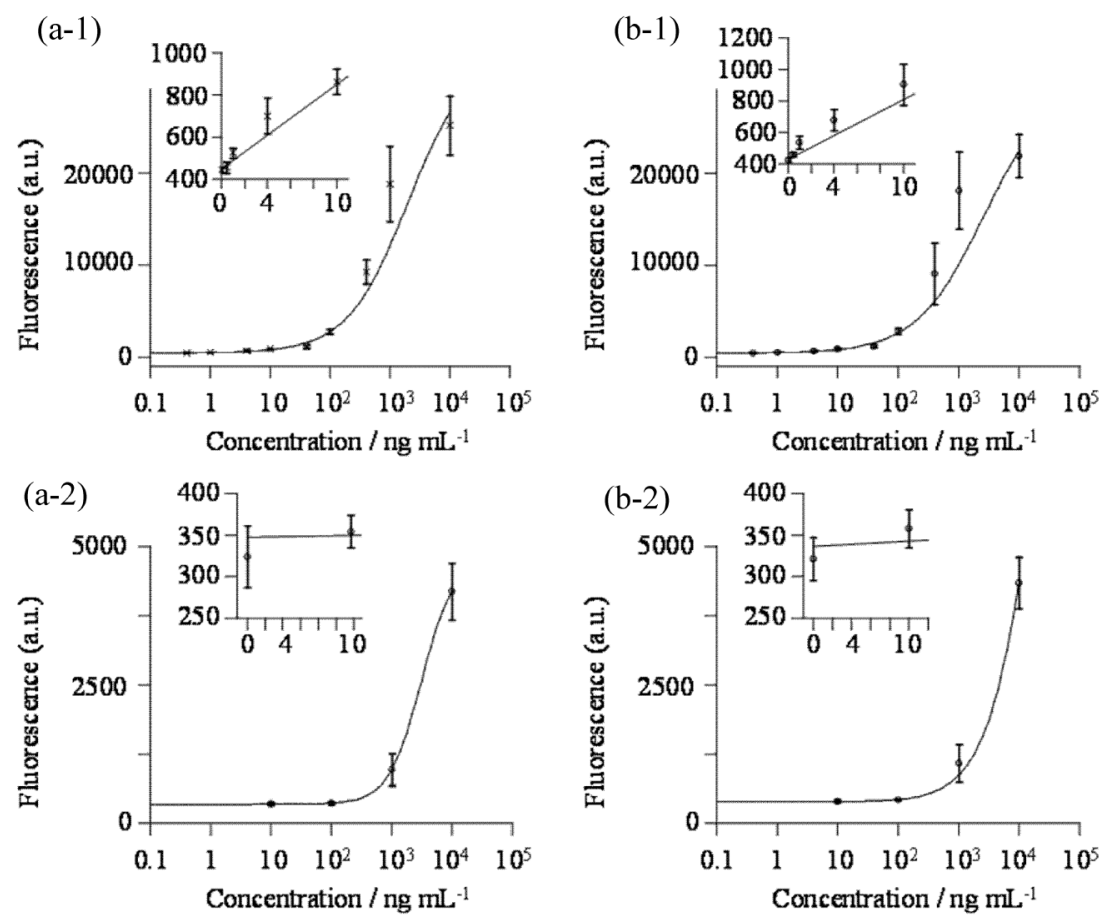

Fig. 3 Calibration curve of PSA diluted in PBS (a-1 and a-2) or human female serum (b-1 and b-2). The error bars represent one standard deviation of four independent experiments. (a-1 or b-1) The curves were obtained from LFDA and the amplification time was $7.0 \mathrm{~min}$. Insets magnify the graphs of the low concentration range. (a-2 and b-2) Calibration curve of PSA without amplification as control experiments. The time of detection was 4.0 min after the start of flow of F-SA.

PSA-spiked PBS and PSA-spiked human female serum. The time courses using the two samples represent much the same performance: not only the curve of the graph but also the fluorescence intensity. These results indicate that the female serum contained no, or a small amount, of PSA below the level of detection, and that component of the serum offered no obvious inhibition of MIA. Higher fluorescent signals were obtained with higher concentrations of PSA. However, a concentration-dependent saturation of fluorescent signal was obtained at high concentrations of PSA, which would be caused by the steric hindrance or trade-off between the fluorescent signal enhancement and quenching. ${ }^{18}$ As a control experiment, no amplification of MIA, described in experimental section, is shown in Figs. 2a-2 and 2b-2. Unlike Figs. 2a-1 and 2b-1, although the graphs were concentration-dependent and no apparent differences of the performance occurred in either PSA-spiked PBS or human female serum, the fluorescent intensities of each sample remained constant over time, and that of $1 \mu \mathrm{g} / \mathrm{mL}$ PSA was lower than LFDA by an order of magnitude $\left(10^{3}\right.$ a.u. to $10^{4}$ a.u.). It was obvious that LFDA definitely contributed to the enhancement of fluorescent signals and the dynamic concentration range.

\section{Calibration and limit of detection ( $L O D)$}

The fluorescent intensity of the blank sample gained significant growth around 7 to $8 \mathrm{~min}$ (Figs. 2a-1 and 2b-1). As described in the experimental section, both the fluorescent intensity and the three standard deviations of a blank sample had a great influence on defining the LOD, and a slower growth gave a better LOD, which meant that the two factors of the blank sample were regarded to be the analytical noise of MIA, and were crucially affected to define LOD. The data of the time course at 7 min amplification time in Figs. $2 a-1$ and $2 b-1$ were plotted to draw dose response curves of PSA diluted in PBS or human female serum (Figs. 3a-1 and 3b-1), and to define the LOD for both samples. As expected from Figs. 2a-1 and 2b-1, the dose response curves of PSA diluted in PBS and human female serum were almost identical in terms of both the dynamic range, five orders of magnitude of PSA concentration, and the LOD. The calculated LOD from Figs. 3a-1 and 3b-1: 490 pg/mL $(15 \mathrm{pM})$ of LOD for PSA-spiked PBS and $520 \mathrm{pg} / \mathrm{mL}(16 \mathrm{pM})$ of LOD for PSA-spiked human female serum. The dynamic range of the PSA concentration also covered the cut-off values $(4-10 \mathrm{ng} / \mathrm{mL}$ ) to distinguish PCa (magnified graphs of Figs. 3a-1 and 3b-1). The coefficients of variation were within $15 \%(n=4)$, except for the data points of 400 and $1000 \mathrm{ng} / \mathrm{mL}$ of PSA-spiked human female serum. This good reproducibility was comparable with results obtained from previously published microfluidic immunoassays..$^{20,21}$

Additionally, the plots of no amplification as control experiments stayed flat and provided a LOD of $270 \mathrm{ng} / \mathrm{mL}$ $(8.2 \mathrm{nM})$ or $140 \mathrm{ng} / \mathrm{mL}(4.2 \mathrm{nM})$, respectively (Figs. 3a-2 and 3b-2). LOD using LFDA was improved by several hundred times compared with the control experiments and there were no obvious differences of the LOD between PSA-spiked PBS and PSA-spiked human female serum, which further validated that the serum sample could be applied to MIA without any harmful effect.

Figure 4 shows the change of the LOD with the passage of the amplification time. The amplification of the fluorescent signal had a time-dependency that also affected the LOD. The plots of PSA-spiked human female serum with LFDA indicated that LOD were improved untill $7 \mathrm{~min}$ of amplification time, but steadily deteriorated from this time (the plots (๑) in Fig. 4), 


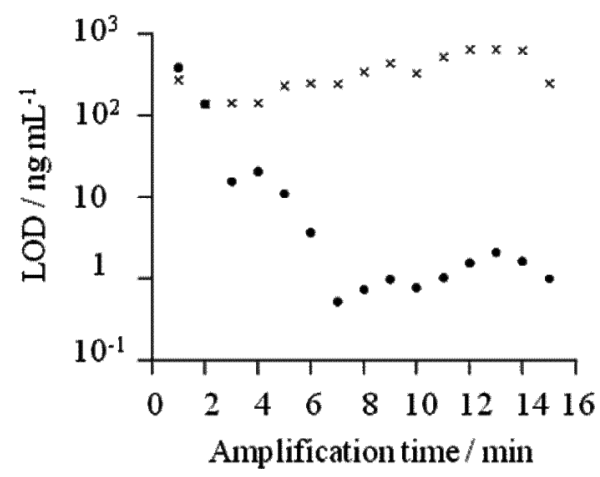

Fig. 4 Plots of the limit of detection over the amplification time under the conditions of LFDA ( ) and control experiments (no amplification) $(\times)$.

whereas a marginal time-dependent variation of the LOD was observed in the plots of control experiments (the plots $(X)$ in Fig. 4) and the LOD of 4 min of amplification time was the best using serum-diluted PSA samples. LFDA has a trade-off between the signal enhancement and non-specific binding of the reagents: b-Ab, F-SA and b-anti-SA. Until $7 \mathrm{~min}$, a fluorescent enhancement from PSA preceded that from the non-specific adsorption, but the latter became comparable to the former at $7 \mathrm{~min}$, which was namely the turning point. On the other hand, under control experiments, only a marginal time-dependent variation was observed because there was no such trade-off. It was perfectly validated that LFDA was responsible for improving the LOD compared with the control experiments.

Previously, MIA for the CRP sample was developed to integrate both power-free microchip and LFDA. ${ }^{18}$ The MIA realized a highly sensitive detection of CRP diluted in CRP-free human serum, and the LOD was $17 \mathrm{pg} / \mathrm{mL}$. Compared with previous results, the LOD of this report was worse by two orders of magnitudes, although the experimental condition was similar, except for the sample. We speculate that this difference might have been caused by the differences in the affinity and the specificity of the antibodies that we used.

\section{Conclusions}

MIA of PSA diluted in human serum was performed with two original technologies: power-free microchip and LFDA. Sequential injection of the reagents for MIA and rapid immunoreactions were achieved with these technologies. The assay was finished within $21 \mathrm{~min}$ (5 min of c-Ab immobilization, $4 \mathrm{~min}$ of blocking, $5 \mathrm{~min}$ of sandwich immunocomplex formation and 7 min of LFDA) with a sample volume of $500 \mathrm{~nL}$. High sensitivity $(520 \mathrm{pg} / \mathrm{mL}, 16 \mathrm{pM}$ covering the cutoff values: 4-10 ng/mL) and quantitative analysis were realized. Additionally, LFDA dramatically improved the sensitivity of the microfluidic immunoassay enough to detect the cut-off values of PSA for the diagnosis of PCa. This comes one step closer to achieving POCT. Diagnosis of the recurrent PCa requires a further improved LOD covering $100 \mathrm{pg} / \mathrm{mL}$ or less of PSA in human serum, which is also known as "undetectable PSA". Therefore, future work is to develop new methodologies to be capable of detecting "undetectable PSA".

\section{References}

1. Information for patients, Japanese Foundation for Cancer Research, http://www.jfcr.or.jp/hospital/conference/cancer/ about/prostate.html.

2. A. Jemal, R. Siegel, E. Ward, Y. Hao, J. Xu, T. Murray, and M. J. Thun, CA. Cancer J. Clin., 2008, 58, 71.

3. H. Lilja, D. Ulmert, and A. J. Vickers, Nat. Rev. Cancer, 2008, 8, 268.

4. D. D. Brooks, A. M. D. Wolf, R. A. Smith, C. Dash, and I. Guessous, J. Natl. Med. Assoc., 2010, 102, 423.

5. S. K. Sia and L. J. Kricka, Lab Chip, 2008, 8, 1982.

6. C. Fernández-Sánchez, C. J. McNeil, K. Rawson, O. Nilsson, H. Y. Leung, and V. Gnanapragasam, J. Immunol. Methods, 2005, 307, 1.

7. G. Liu, Y. Y. Lin, J. Wang, H. Wu, C. M. Wai, and Y. Lin, Anal. Chem., 2007, 79, 7644.

8. C. K. Mauck, Sex. Transm. Dis., 2009, 36, S81.

9. S. McWilliams and B. Gartside, J. Forensic Sci., 2009, 54, 610.

10. D. A. Giljohann and C. A. Mirkin, Nature, 2009, 462, 461.

11. Y. J. Ko, J. H. Maeng, Y. Ahn, S. Y. Hwang, N. G. Cho, and S. H. Lee, Electrophoresis, 2008, 29, 3466.

12. B. S. Lee, J. N. Lee, J. M. Park, J. G. Lee, S. Kim, Y. K. Cho, and C. Ko, Lab Chip, 2009, 9, 1548.

13. P. S. Waggoner, M. Varshney, and H. G. Craighead, Lab Chip, 2009, 9, 3095.

14. L. Gervais and E. Delamarche, Lab Chip, 2009, 9, 3330.

15. M. Ikami, A. Kawakami, M. Kakuta, Y. Okamoto, N. Kaji, M. Tokeshi, and Y. Baba, Lab Chip, 2010, 10, 3335.

16. K. Hosokawa, K. Sato, N. Ichikawa, and M. Maeda, Lab Chip, 2004, 4, 181.

17. K. Hosokawa, M. Omata, K. Sato, and M. Maeda, Lab Chip, 2006, 6, 236.

18. K. Hosokawa, M. Omata, and M. Maeda, Anal. Chem., 2007, 79, 6000 .

19. T. K. Christopoulos and E. P. Diamandis, in "Immunoassay", ed. E. P. Diamandis and T. K. Christopoulos, 1996, Academic Press, San Diego, 25 - 50.

20. J. Ziegler, M. Zimmermann, P. Hunziker, and E. Delamarche, Anal. Chem., 2008, 80, 1763.

21. C. Jönsson, M. Aronsson, G. Rundström, C. Pettersson, I. Mendel-Hartvig, J. Bakker, E. Martinsson, B. Liedberg, B. MacCraith, O. Ohman, and J. Melin, Lab Chip, 2008, 8, 1191. 